\title{
Erratum to: Locating foreign aid commitments in response to political violence
}

\author{
Paul Bezerra $^{1}$ - Alex Braithwaite ${ }^{1}$
}

Published online: 1 November 2016

(C) Springer Science+Business Media New York 2016

\section{Erratum to: Public Choice DOI 10.1007/s11127-016-0377-9}

Due to an unfortunate combination of error and oversight on the part of Braithwaite, Tables 2, 3 and 4 in the original published version contain a considerable number of erroneous attributions of statistical significance to coefficient estimates. This error occurred at some stage between acceptance of the manuscript and final publication. Please find in this erratum the correct version of the tables that should be regarded as the final version by the reader. Please also note that all of the coefficient values and standard errors are reported correctly in the original version.

The online version of the original article can be found under doi:10.1007/s11127-016-0377-9.

$\triangle$ Alex Braithwaite

abraith@email.arizona.edu

1 School of Government and Public Policy, University of Arizona, Tucson, USA 
Table 2 Zero-inflated negative binomial regression models for conflict, aid, and terrorism

\begin{tabular}{|c|c|c|c|}
\hline & Terrorism model & Conflict model & Combined model \\
\hline \multicolumn{4}{|l|}{ Count model } \\
\hline 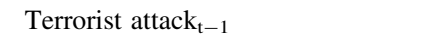 & $0.541 *(0.224)$ & - & $0.503 *(0.233)$ \\
\hline State-based conflict $t_{t-1}$ & - & $0.302 *(0.118)$ & $0.271 *(0.120)$ \\
\hline $\begin{array}{l}\text { Proportion of neighbors } \\
\text { experiencing } \text { conflict }_{t-1}\end{array}$ & $0.032(0.135)$ & $-0.016(0.142)$ & $-0.008(0.142)$ \\
\hline $\ln (\text { gross cell product per capita })_{t-1}$ & $0.189 *(0.074)$ & $0.188 *(0.074)$ & $0.186 *(0.074)$ \\
\hline Human footprint (mean) & $-0.002(0.009)$ & $-0.0004(0.009)$ & $-0.001(0.009)$ \\
\hline \# Aid commitments $\mathrm{t}_{-1}$ & $0.206 * * *(0.025)$ & $0.208 * * *(0.024)$ & $0.206 * * *(0.025)$ \\
\hline Peace area & $0.237(0.193)$ & $-0.019(0.193)$ & $0.005(0.196)$ \\
\hline Number of neighbors & $0.136^{\dagger}(0.076)$ & $0.125^{\dagger}(0.073)$ & $0.132^{\dagger}(0.072)$ \\
\hline $\ln ($ distance to border) & $-0.065^{\dagger}(0.036)$ & $-0.058(0.037)$ & $-0.057(0.036)$ \\
\hline $\ln ($ distance to capital $)$ & $0.029(0.056)$ & $0.027(0.055)$ & $0.035(0.056)$ \\
\hline Constant & $-2.283 *(1.103)$ & $-2.249 *(1.081)$ & $-2.328 *(1.077)$ \\
\hline \multicolumn{4}{|l|}{ Inflate model } \\
\hline 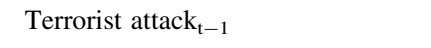 & $-1.069(0.696)$ & - & $-0.702(0.722)$ \\
\hline State-based conflict $t_{t-1}$ & - & $-1.417 * * *(0.274)$ & $-1.403 * * *(0.279)$ \\
\hline $\begin{array}{l}\text { Proportion of neighbors } \\
\text { experiencing } \text { conflict }_{t-1}\end{array}$ & $0.065(0.126)$ & $0.100(0.129)$ & $0.106(0.129)$ \\
\hline $\ln (\text { gross cell product per capita })_{t-1}$ & $0.579 * * *(0.093)$ & $0.590 * * *(0.092)$ & $0.588 * * *(0.093)$ \\
\hline Human footprint (mean) & $-0.087 * * *(0.008)$ & $-0.086 * * *(0.008)$ & $-0.086 * * *(0.008)$ \\
\hline \# Aid commitments $\mathrm{t}_{-1}$ & $-4.153 * * *(0.337)$ & $-4.194 * * *(0.322)$ & $-4.172 * * *(0.321)$ \\
\hline Peace area & $-0.942 * * *(0.207)$ & $0.414(0.312)$ & $0.408(0.318)$ \\
\hline Number of neighbors & $-0.019(0.100)$ & $-0.002(0.101)$ & $0.002(0.101)$ \\
\hline $\ln ($ distance to border) & $0.161 * * *(0.041)$ & $0.149 * * *(0.042)$ & $0.148 * * *(0.042)$ \\
\hline $\ln ($ distance to capital $)$ & $0.379 * * *(0.072)$ & $0.353 * * *(0.072)$ & $0.353 * * *(0.072)$ \\
\hline Constant & $-2.159^{\dagger}(1.233)$ & $-2.165^{\dagger}(1.244)$ & $-2.165^{\dagger}(1.248)$ \\
\hline Observations & 41,331 & 41,331 & 41,331 \\
\hline AIC & $20,382.7$ & $20,326.6$ & $20,318.4$ \\
\hline
\end{tabular}

Robust standard errors clustered on grid-cell reported in parentheses

${ }^{\dagger} p<.10 ; * p<.05 ; * * p<.01 ; * * * p<.001$

Table 3 Zero-inflated negative binomial regression models of time since attack

Terror \& time since attack model

$\begin{array}{ll}\text { Count model } & \\ \text { Time since attack } & 0.268^{* *}(0.095) \\ \text { Time since attack }^{2} & -0.034(0.022) \\ \text { Time since attack } & \\ \text { Proportion of neighbors } & 0.001(0.001) \\ \quad \text { experiencing conflict }{ }_{\mathrm{t}-1} & 0.016(0.137) \\ \text { ln(gross cell product per capita) })_{\mathrm{t}-1} & \\ \text { Human footprint (mean) } & 0.181(0.075) \\ \text { \# Aid commitments } \mathrm{t}_{\mathrm{t}-1} & -0.004(0.009) \\ \end{array}$

Conflict \& time since attack model
$0.397 * * *(0.102)$

$0.057 *(0.024)$

$0.002(0.001)$

$0.029(0.135)$

$0.156 *(0.068)$

$-0.001(0.010)$

$0.193 * * *(0.025)$ 
Table 3 continued

\begin{tabular}{|c|c|c|}
\hline & $\begin{array}{l}\text { Terror \& time since } \\
\text { attack model }\end{array}$ & $\begin{array}{l}\text { Conflict \& time since } \\
\text { attack model }\end{array}$ \\
\hline Peace area & $0.202(0.197)$ & $-0.059(0.204)$ \\
\hline Number of neighbors & $0.126(0.078)$ & $0.124(0.079)$ \\
\hline $\ln$ (distance to border) & $-0.063^{\dagger}(0.037)$ & $-0.023(0.036)$ \\
\hline $\ln$ (distance to capital) & $0.043(0.056)$ & $-0.013(0.055)$ \\
\hline Constant & $-2.211^{*}(1.127)$ & $-2.173^{\dagger}(1.136)$ \\
\hline \multicolumn{3}{|l|}{ Inflate model } \\
\hline Time since attack & $-0.619 *(0.289)$ & $-0.522 *(0.205)$ \\
\hline Time since attack ${ }^{2}$ & $0.086(0.056)$ & $0.079(0.055)$ \\
\hline Time since attack ${ }^{3}$ & $-0.003(0.003)$ & $-0.003(0.003)$ \\
\hline $\begin{array}{l}\text { Proportion of neighbors } \\
\text { experiencing conflict } t_{t-1}\end{array}$ & $0.085(0.128)$ & $0.202(0.134)$ \\
\hline $\ln (\text { gross cell product per capita })_{t-1}$ & $0.570 * * *(0.094)$ & $0.626^{* * *}(0.091)$ \\
\hline Human footprint (mean) & $-0.088 * * *(0.008)$ & $-0.076^{* * *}(0.009)$ \\
\hline \# Aid commitments $\mathrm{s}_{\mathrm{t}-1}$ & $-4.175^{* * *}(0.342)$ & $-4.108 * * *(0.341)$ \\
\hline Peace area & $-0.930 * * *(0.214)$ & $-0.336(0.253)$ \\
\hline Number of neighbors & $-0.019(0.101)$ & $-0.020(0.107)$ \\
\hline $\ln ($ distance to border) & $0.147 * * *(0.043)$ & $0.150 * * *(0.045)$ \\
\hline $\ln$ (distance to capital) & $0.362 * * *(0.073)$ & $0.400 * * *(0.073)$ \\
\hline Constant & $-1.899(1.250)$ & $-2.759 *(1.266)$ \\
\hline Observations & 41,331 & 41,331 \\
\hline AIC & $20,335.7$ & $20,079.2$ \\
\hline
\end{tabular}

Robust standard errors clustered on grid-cell reported in parentheses

${ }^{\dagger} p<.10 ; * p<.05 ; * * p<.01 ; * * * p<.001$

Table 4 Zero-inflated negative binomial regression models of severity and aid

\begin{tabular}{|c|c|c|}
\hline & Terror \& severity model & Conflict \& severity model \\
\hline \multicolumn{3}{|l|}{ Count model } \\
\hline Attack $_{\mathrm{t}-1}$ & $0.654 * *(0.220)$ & $0.279 *(0.123)$ \\
\hline Attack severity ${ }_{t-1}$ & $-0.035^{* *}(0.011)$ & $0.0003(0.0004)$ \\
\hline Attack severity $y_{t-1}^{2}$ & $0.0002^{* *}(0.0001)$ & $-8.43^{\mathrm{e}-8}\left(6.27^{\mathrm{e}-8}\right)$ \\
\hline $\begin{array}{l}\text { Proportion of neighbors } \\
\text { experiencing conflict } t_{t-1}\end{array}$ & $0.035(0.135)$ & $-0.015(0.142)$ \\
\hline $\ln (\text { gross cell product per capita })_{t-1}$ & $0.188 *(0.074)$ & $0.192 *(0.075)$ \\
\hline Human footprint (mean) & $-0.002(0.009)$ & $-0.0001(0.009)$ \\
\hline \# Aid commitments $\mathrm{t}_{-1}$ & $0.207 * * *(0.025)$ & $0.208 * * *(0.234)$ \\
\hline Peace area & $0.245(0.193)$ & $-0.018(0.192)$ \\
\hline Number of neighbors & $0.139^{\dagger}(0.075)$ & $0.132^{\dagger}(0.072)$ \\
\hline $\ln ($ distance to border $)$ & $-0.064^{\dagger}(0.036)$ & $-0.057(0.037)$ \\
\hline $\ln ($ distance to capital) & $0.029(0.056)$ & $0.030(0.056)$ \\
\hline Constant & $-2.304 *(1.102)$ & $-2.364 *(1.101)$ \\
\hline
\end{tabular}


Table 4 continued

\begin{tabular}{|c|c|c|}
\hline & Terror \& severity model & Conflict \& severity model \\
\hline \multicolumn{3}{|l|}{ Inflate model } \\
\hline Attack $_{\mathrm{t}-1}$ & $-1.034(0.808)$ & $-1.471 * * *(0.286)$ \\
\hline Attack severity $_{t-1}$ & $0.726(0.714)$ & $0.001(0.001)$ \\
\hline Attack severity ${ }_{t-1}^{2}$ & $-0.083(0.057)$ & $-2.33^{\mathrm{e}-7 \dagger}\left(1.20^{\mathrm{e}-7}\right)$ \\
\hline $\begin{array}{l}\text { Proportion of neighbors } \\
\text { experiencing } \text { conflict }_{t-1}\end{array}$ & $0.070(0.126)$ & $0.100(0.129)$ \\
\hline $\ln (\text { gross cell product per capita })_{t-1}$ & $0.580 * * *(0.093)$ & $0.596 * * *(0.093)$ \\
\hline Human footprint (mean) & $-0.087 * * *(0.008)$ & $-0.086^{* * *}(0.008)$ \\
\hline$\#$ Aid commitments $\mathrm{t}_{-1}$ & $-4.152 * * *(0.335)$ & $-4.190 * * *(0.320)$ \\
\hline Peace area & $-0.937 * * *(0.207)$ & $0.411(0.315)$ \\
\hline Number of neighbors & $-0.014(0.100)$ & $0.005(0.101)$ \\
\hline $\ln ($ distance to border) & $0.162 * * *(0.041)$ & $0.151 * * *(0.042)$ \\
\hline $\ln ($ distance to capital) & $0.379 * * *(0.072)$ & $0.357 * * *(0.072)$ \\
\hline Constant & $-2.200^{\dagger}(1.231)$ & $-2.289^{\dagger}(1.255)$ \\
\hline Observations & 41,331 & 41,331 \\
\hline AIC & $20,381.8$ & $20,329.2$ \\
\hline
\end{tabular}

Robust standard errors clustered on grid-cell reported in parentheses

${ }^{\dagger} p<.10 ; * p<.05 ; * * p<.01 ; * * * p<.001$ 